\title{
Spectroscopie et cinétique des gaz rares et leurs mélanges sous excitation multiphotonique
}

\author{
Y. Salamero et P. Millet
}

Laboratoire de Physique des Plasmas et de leurs Applications de Toulouse, ESA 5002,

Université P. Sabatier, 118 route de Narbonne, 31062 Toulouse cedex, France

\begin{abstract}
The purpose of this work is to bring out the analogous behaviour of krypton and xenon under multiphotonic excitation through three photons absorption. Excitation is acheived either on the ${ }^{3} \mathrm{P}_{1}$ state itself or in its neighbourhood. In this latter case the excitation consists of populating the dissociative $1_{u}\left({ }^{3} \mathrm{P}_{1}\right)$ molecular state from ground state dimers. It also appears that, in our experimental conditions, excitation is mainly achieved through absorption of a VUV photon originating from the third harmonic of the incident laser beam. In the $\mathrm{Kr}$-Xe mixture our excitation spectra bear both krypton and xenon components. The temporal analysis of krypton's emission filtered around $128 \mathrm{~nm}$ shows the existence of an energy transfer from the $\mathrm{Kr}\left({ }^{3} \mathrm{P}_{1}\right)$ state towards a $5 \mathrm{~d}$ xenon state through binary collisions whose collision rate is $6,810^{5}$ Torr $^{-1} \mathrm{~s}^{-1}$.
\end{abstract}

\section{I- INTRODUCTION}

Les gaz rares de l'air et leurs mélanges ont fait l'objet ces vingt dernières années de nombreuses études spectroscopiques et cinétiques [1-4] avec des moyens d'excitation forts différents. Pour ce qui nous concerne, les travaux que nous avons réalisés, d'abord avec de particules alpha[5],puis au moyen d'un faisceau laser pulsé et accordable[6] ont pour objectif de déterminer les processus réactionnels responsables de la création et de la disparition des espèces excitées qui conduisent aux émissions U.V.L. des gaz rares. Ces études permettent de mieux cerner les mécanismes réactionnels qui interviennent dans les lasers à excimères dont le développement a été à l'origine de ces multiples études. Les sources incohérentes U.V.L. à décharges à barrières diélectriques qui se développent actuellement constituent un champ d'application supplémentaire.

Le but de cette expérience est de réaliser des études spectroscopiques et cinétiques en procédant à une excitation sélective. Etant donné l'énergie élevée des premiers états excités des gaz rares nous nous sommes orientés vers une excitation par absorption multiphotoni-que. Notre source d'excitation permet d'atteindre les états résonnants ${ }^{3} P_{1}$ et ${ }^{1} \mathrm{P}_{1}$ des gaz rares les plus lourds par absorption de trois photons. Les règles de sélection prévoient que ce type de transition est permis et de ce fait la probabilité de peupler un des états résonnants n'est pas négligeable sous certaines conditions. La mise en évidence nécessite un flux lumineux important que l'on obtient au moyen d'un faisceau laser focalisé dans le gaz.

Le dispositf expérimental, a été décrit dans des publications antérieures[7]. II comporte un laser à colorant pompé par un laser à azote qui délivre des impulsions de 5ns toutes les $20 \mathrm{~ms}$. Ce faiceau est accordable dans le domaine $360-375 \mathrm{~nm}$ avec une largeur spectrale voisine de $1 \mathrm{~cm}^{-1}$.Il est focalisé au centre d'une cellule en acier inoxydable par une lentille de courte focale. La densité d'énergie au niveau du point focal est de l'ordre du GW par $\mathrm{cm}^{2}$. La luminescence provenant de la désexcitation du gaz est détectée grâce à d'un 
photomulitiplicateur EMR $590 \mathrm{G}$ fonctionnant en régime de détection par photons isolés. La distribution spectrale de l'émission lumineuse a été tracée dans le domaine de l'ultraviolet lointain au moyen d'un monochromateur fonctionnant sous vide.. Une émission peut être sélectionnée par un filtre interférentiel UVL pour suivre son évolution au cours du temps ou enregistrer son spectre d'excitation. Le domaine de pressions couvert dans les gaz est compris entre $10^{-2}$ et 750 Torr. Dans le mélange $\mathrm{Kr}$-Xe le krypton constitue le gaz majoritaire et la pression totale peut varier de quelques dizaines de Torr à la pression atmosphérique .

Nous étudions à l'heure actuelle le transfert d'énergie dans le mélange $\mathrm{Kr}$-Xe. Avant d'aborder l'étude du mélange krypton-xénon, il est intéressant de comparer les résultats obtenus dans le xénon et le krypton purs.

\section{I-RESULTATS SPECTROSCOPIQUES DANS le KRYPTON et le XENON PURS}

Aux faibles pressions de remplissage ( de $10^{-2}$ Torr à quelques Torr) les seules émissions décelables sont les raies de résonance situées à $123,6 \mathrm{~nm}$ pour le krypton et 146,9 $\mathrm{nm}$ pour le xénon.. On peut voir sur les figures 1 -a et 1-b les spectres obtenus respectivement dans le xénon et le krypton purs lorsque l'excitation porte sur l'état résonnant ${ }^{3} \mathrm{P}_{1}$ de ces gaz. La faible largeur de ces spectres montre bien, qu'à ces pressions, l'excitation résonnante à trois photons est principalement de type atomique. Nous avons vérifié expérimentalement que la loi d'évolution de l'intensité de la luminescence en fonction de la puissance $P_{1}$ du faisceau laser incident était bien une loi en $\mathrm{P}_{1}^{3}$.
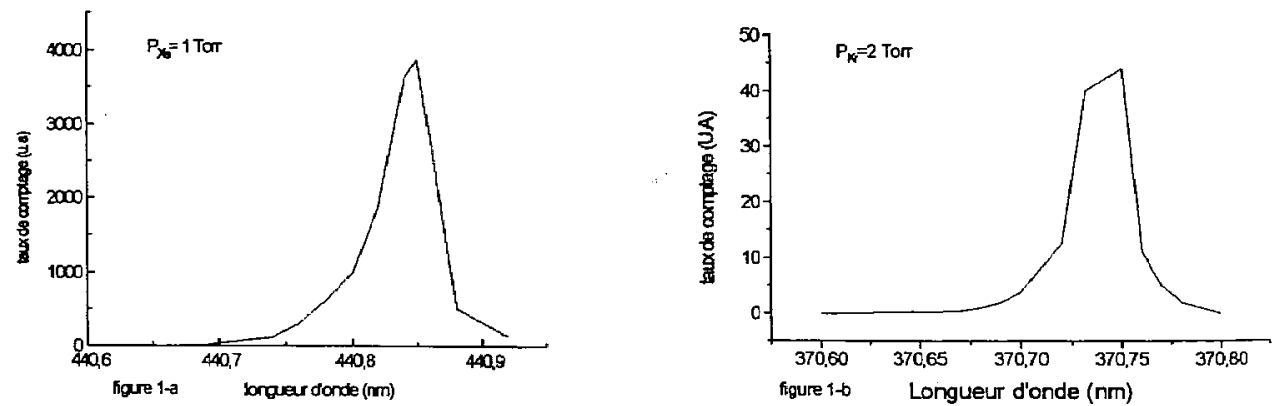

Figures 1-a et 1-b: Spectres d'excitation multiphotonique du krypton et du xénon à faible pression

La section efficace d'absorption multiphotonique à trois photons pour la transition ${ }^{3} \mathrm{P}_{1^{-}}{ }^{1} \mathrm{~S}_{0}$ a été estimée : elle est de l'ordre de $10^{-81} \mathrm{~cm}^{6} \mathrm{~s}-1$ pour le xénon et le krypton. Une dissymétrie des spectres d'excitation qui va s'accentuant avec l'augmentation de pression traduit la formation des dimères de gaz rares à l'état fondamental $0_{\mathrm{g}}{ }^{+}$.

Lorsque la pression est supérieure à quelques Torr, il se produit une excitation de type moléculaire. Dans le xénon et le krypton purs, les spectres d'excitation s'élargissent et se décalent du côté des courtes longueurs d'onde, figures 2-a et 2-b. L'interprétation de ce phénomène repose sur la présence de paires libres et liées comme l'ont démontré Freeman et al [8] et M.C. Castex [9] dans des expériences d'absorption au voisinage de la raie de résonance du xénon. Les niveaux de vibration élevées de la molécule à l'état fondamental $\mathrm{Og}+$ sont peuplés de façon non négligeable par rapport au niveau $\mathrm{v}=0$ 
car le puits de potentiel du dimère est de l'ordre de l'énergie d'agitation thermique à température ambiante: $192 \mathrm{~cm}^{-1}$ pour le xénon et et $137 \mathrm{~cm}^{-1}$ pour le krypton.

Les positions relatives de courbes de potentiels des états moléculaires les plus bas, permettent de déduire que l'on excite l'état moléculaire dissociatif $1 \mathrm{u}\left({ }^{3} \mathrm{P}_{1}\right)$.

Les émissions U.V.L. observées sont les émissions caractéristiques du krypton et du xénon. A partir d'une dizaine de Torr le premier continuum est observable dans le krypton et dans le xénon. Lorsque la pression du gaz augmente, le comportement est identique dans les deux gaz: le deuxième continuum apparaît (centré à $145 \mathrm{~nm}$ pour le krypton et $173 \mathrm{~nm}$ pour le xénon ) au détriment du premier. Aux très fortes pressions (quelques centaines de Torr ) seul ce deuxième continuum est visible dans chacun des gaz.

Le décalage du maximum du spectre d'excitation vers les courtes longueurs d'onde pour des pressions croissantes s'explique par une modification du processus d'excitation. En effet, nous avons mis en évidence dans le cas de l'étude sur le xénon pur la présence de l'harmonique trois du faisceau laser incident dans la zone de dispersion négative (140-147 $\mathrm{nm}$ ) corrélée à la raie de résonance[10]. La focalisation du faisceau au centre de la cellule d'analyse et le défaut de phase dans la zone de dispersion négative au voisinage des raies de résonance nous placent dans des conditions optimales pour la génération de l'harmonique trois.
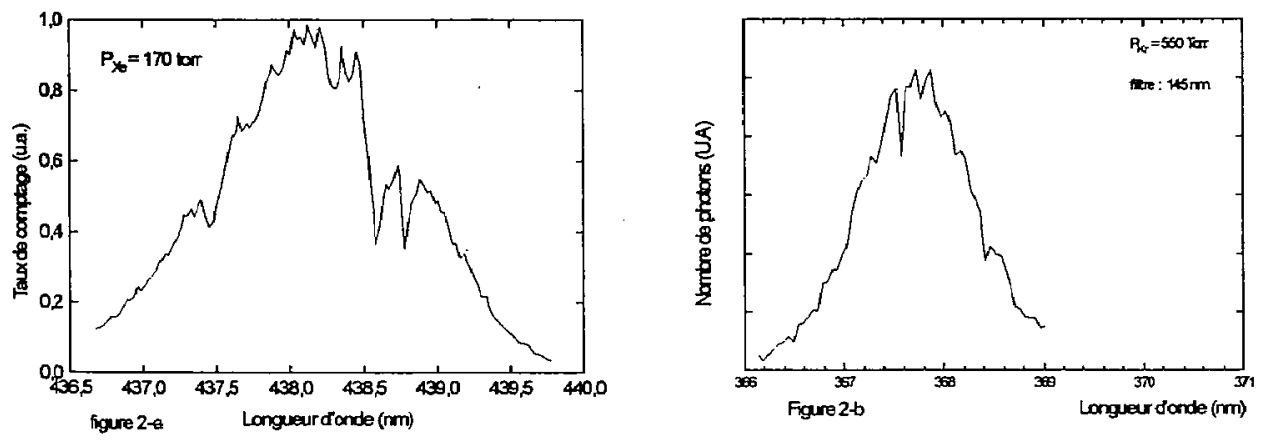

Figures 2-a et 2-b: Spectres d'excitation du krypton et du xénon en luminescence en luminescence filtrée à 173 et $145 \mathrm{~nm}$

Ce phénomène survient également dans le krypton de façon similaire dans le domaine de longueur d'onde correspondant à la zone de dispersion négative (120-123,6 nm) qui jouxte la raie de résonance située à $123,6 \mathrm{~nm}$. Si on se reporte aux travaux réalisés sur ce même dispositif expérimental dans le xénon pur [10] on constate expérimentalement que, lorsque la pression augmente, le maximum de la puissance générée sur l'harmonique trois se produit pour des longueurs d'onde de plus en plus courtes. Ce résultat est analogue dans le krypton pur conformément aux travaux de Mahon et al [11]. Ce résultat est théoriquement prévisible.

Dans le cas d'un faisceau gaussien focalisé par une lentille cette puissance générée sur l'harmonique trois $P_{3 \omega}$ a pour expression :

$$
P_{3 \omega}=\frac{1}{c^{2}} \frac{k_{03}^{4} k_{1}^{2}}{6 k_{3}^{2}} P_{1}^{3} \chi^{(3)^{2}} N^{2} \mathrm{~F}
$$

avec les valeurs des vecteurs d'onde: 


$$
\mathrm{k}_{03}=\frac{2 \pi}{\lambda_{03}} ; \quad \mathrm{k}_{1}=\frac{2 \pi}{\lambda_{1}}=\frac{2 \pi}{3 \lambda_{03}} \mathrm{n}_{1} \quad \text { et } \quad \mathrm{k}_{3}=\frac{2 \pi}{\lambda_{3}}=\frac{2 \pi}{\lambda_{03}} \mathrm{n}_{3}
$$

$-P_{1}$ représente la puissance du faisceau laser incident

Dans nos conditions de focalisation ( le faisceau est focalisé au milieu de la cellule), le produit $\mathrm{N}^{2} \mathrm{~F}$ conditionne la puissance sur l'harmonique trois et s'écrit [13] :

$$
\mathrm{N}^{2} \mathrm{~F}=\frac{\pi}{\left(\mathrm{bC}_{\mathrm{Kr}}\left(\lambda_{03}\right)\right)^{2}}(\mathrm{~b} \Delta \mathrm{k})^{4} \exp (\mathrm{b} \Delta \mathrm{k}) \text { pour } \Delta \mathrm{k}<0 ;
$$

- $b$ désigne le paramètre confocal

$\Delta k=k_{3}-3 k_{1}=\frac{2 \pi}{\lambda_{03}}\left(n_{3}-n_{1}\right)$ est le défaut de phase pour les pulsations $\omega$ et $3 \omega$.

$-\mathrm{C}_{\mathrm{Kr}}$ est le défaut de phase par atome $\left(\mathrm{C}_{\mathrm{Kr}}=\Delta \mathrm{k} / \mathrm{N}\right)$

-pour $\Delta \mathrm{k} \geq 0$, la fonction $F$ est nulle.

- pour $\Delta k<0$ on montre que le produit $\mathrm{N}^{2} \mathrm{~F}$ est maximum lorsque le produit

b. $\Delta \mathbf{k}=-4$. Cela survient, dans un gaz pur à une pression bien déterminée pour une longueur d'onde donnée. Par exemple dans le xénon, pour une longueur d'onde d'excitation de $435,6 \mathrm{~nm}$ la pression optimale correspondante est de 250 Torr.

Le peuplement de l'état moléculaire dissociatif $l u\left({ }^{3} \mathrm{P}_{1}\right)$ peut donc survenir par absorption multiphotonique et par absorption d'un photon UVL provenant de l'harmonique trois. Les deux processus conduisent,après dissociation rapide de l'état moléculaire $1 \mathrm{u}\left({ }^{3} \mathrm{P}_{1}\right)$, à l'état résonnant ${ }^{3} \mathbf{P}_{1}$.

On constate également des défauts de luminescence sur les spectres d'excitation qui se produisent pour certaines longueurs d'onde du faisceau laser. Ils peuvent être attribués à une ionisation multiphotonique du gaz à certaines longueurs d'onde d'excitation. En effet, lorsque l'énergie du photon incident est résonnante à trois ou à quatre photons avec un état excité supérieur à la configuration $5 \mathrm{~s}, 5 \mathrm{~s}^{\prime}$ pour le krypton ou $6 \mathrm{~s}, 6 \mathrm{~s}^{\prime}$ pour le xénon, il est possible d'ioniser le gaz par absorption de photons supplémentaires depuis cet état. La sélectivité recherchée est alors perdue. Afin de contrôler ce processus, nous avons mis au point un dispositif de détection des photoélectrons [7]. Un champ électrique de $40 \mathrm{~V} / \mathrm{cm}$ est appliqué au niveau du point focal et les charges sont collectées par les armatures d'un condensateur plan. La mesure est effectuée au moyen d'un électromètre Keithley dont le courant de bruit est voisin de $0,1 \mathrm{fA}$.

A titre d'exemple, on trouvera figure 3 le spectre d'ionisation obtenu pour une pression partielle de krypton de 200 Torr avec $1 \%$ de xénon. Les pics d'ionisation notées $\lambda_{3}$ et $\lambda_{12}$ correspondent à l'ionisation du xénon par absorption de trois photons à partir de l'état fondamental Xe $\left({ }^{1} \mathrm{~S}_{0}\right)$ via les états $\mathrm{Xe} 5 \mathrm{~d}[5 / 2]_{\mathrm{J}=3}$. et $\mathrm{Xe} 5 \mathrm{~d}[7 / 2]_{\mathrm{J}=3}$. L'absorption d'un photon supplémentaire depuis ces états conduit au continuum d'ionisation.

Les pics d'ionisation aux longueurs d'onde $\lambda_{9}, \lambda_{10}$ et $\lambda_{11}$ sont attribués à l'ionisation du krypton via les états $6 p^{\prime}[1 / 2]_{\mathrm{J}=0}, 6 \mathrm{p}^{\prime}[3 / 2]_{\mathrm{J}=0}$ et $5 \mathrm{f}[3 / 2]_{\mathrm{J}=2}$ par des processus à $3+1$ et $4+1$ photons. Comme on peut le constater sur ce spectre d'ionisation, il existe une composante continue de très faible amplitude comprise entre 360 et $370 \mathrm{~nm}$, analogue à celle des spectres d'excitation dans le krypton pur.. Cette composante traduit l'existence d'une très faible ionisation par absorption de deux photons supplémentaires depuis l'état $\operatorname{Kr}\left({ }^{3} \mathrm{P}_{1}\right)$ obtenu par dissociation de l'état lu. 


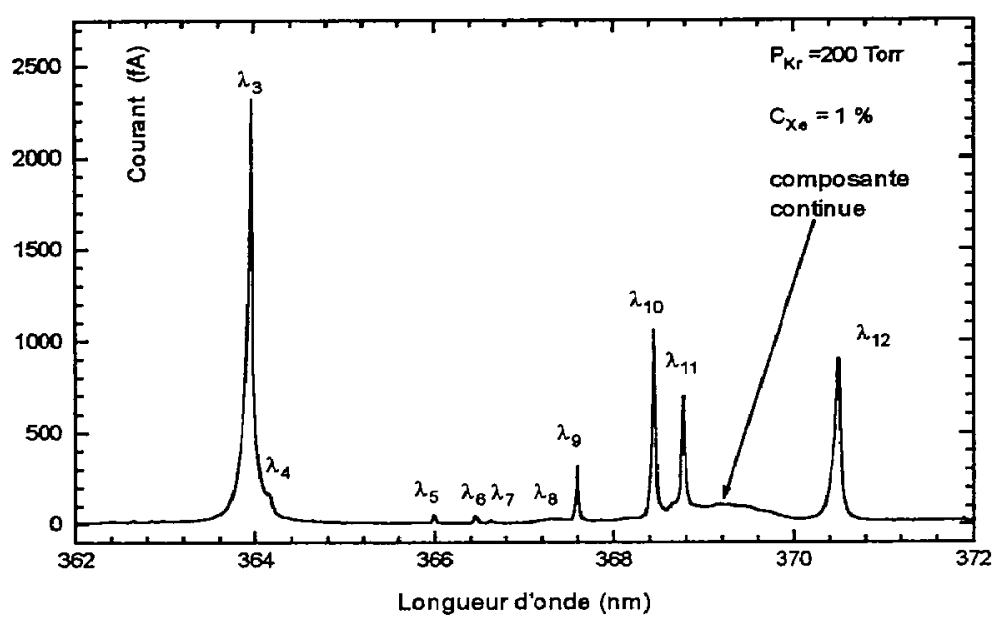

figure3: spectres d'ionisation multiphotonique dans le mélange $\mathrm{Kr}-\mathrm{Xe}$

Ces résultats nous ont permis de nous placer,pour les études cinétiques, dans des conditions d'excitation où le nombre d'ions produits par impulsion laser est faible par rapport au nombre d'états résonnants ${ }^{3} \mathrm{P}_{1}$.

\section{III- ETUDE DU MELANGE Kr-Xe}

Bien que l'excitation se produise initialement sur l'état moléculaire $\mathrm{Kr}_{2}\left[1 \mathrm{u}\left({ }^{3} \mathrm{P}_{1}\right)\right]$, les spectres d'émission dans le mélange $\mathrm{Kr}-\mathrm{Xe}$, montrent la présence des émissions du xénon: premier et deuxième continua à 145 et $180 \mathrm{~nm}$ (figure 4 ).

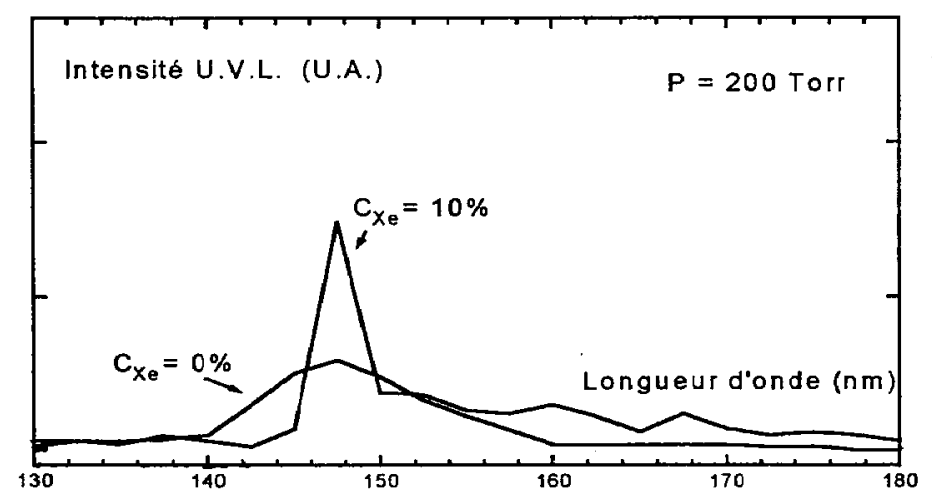

Figure 4: Spectres d'émission consécutifs à une excitation de l'état $\mathrm{Kr}_{2}\left[1 \mathrm{u}\left({ }^{3} \mathrm{P}_{1}\right)\right]$ 
Nous nous sommes assurés que l'excitation portait bien sur le krypton car dans le xénon pur: aucune émission du xénon n'est alors détectée. Pour toutes les émissions observées dans le mélange l'excitation initiale est produite sur l'état $\mathrm{K}_{2}\left[\mathrm{lu}\left({ }^{3} \mathrm{P}_{1}\right)\right]$ comme on peut le contrôler sur les spectres d'excitation.

Par exemple on peut voir à faible pression de remplissage, figures 5-a et 5-b, que la contribution aux émissions situées à 128 et $145 \mathrm{~nm}$ peut se faire également par l'intermédiaire de l'excitation directe de l'état $\mathrm{Xe} 5 \mathrm{~d}[7 / 2]_{\mathrm{J}=3}$ comme en témoigne la présence d'un pic à $370,45 \mathrm{~nm}$. La composante continue, comme dans le krypton pur, se décale du côté des courtes longueurs d'onde lorsque la pression augmente. Les spectres d'excitation et d'ionisation ont un comportement similaire en fonction de la pression de krypton.
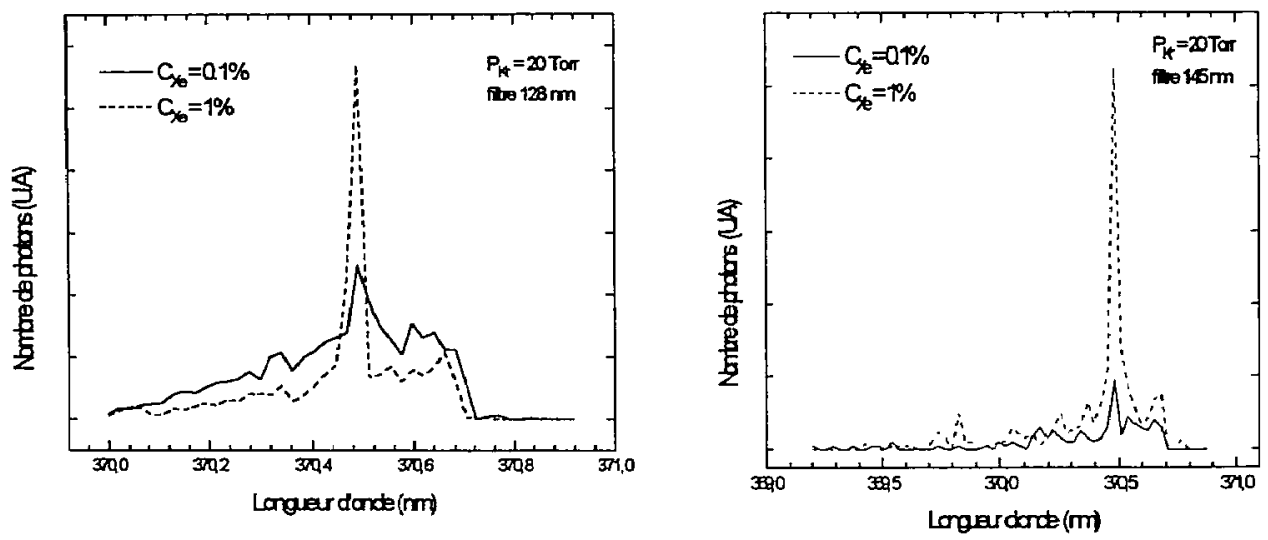

Figure 5 : Spectres d'excitation en luminescence filtrée à $128 \mathrm{~nm}$.et $145 \mathrm{~nm}$ pour $P_{\mathrm{Kr}}=20$ Torr et à différentes concentrations de xénon obtenus

\section{ANALYSE TEMPORELLE à $128 \mathrm{~nm}$ dans le KRYPTON PUR et dans le MELANGE Kr-Xe}

Elle a été menée dans les conditions décrites ci-dessus quant à l'excitation. La luminescence est observée au travers d'un filtre interférentiel centré à $128 \mathrm{~nm}$. Les histogrammes représentatifs de l'évolution temporelle de la luminescence sont enregistrés au moyen d'un analyseur multiéchelle résolu en temps. Un photodiode rapide placée sur la trajectoire du faisceau laser définit l'instant origine. Les impulsions anodiques issues du photomultiplicateur fonctionnant en régime de comptage de photons, après mise en forme, sont classées en fonction du temps.. La résolution de l'appareil est de $5 \mathrm{~ns}$ et le nombre de canaux est égal à 16384 .

Dans le krypton pur le domaine de pression couvert va de 1 à 70 Torr. Les histogrammes qui traduisent le déclin de cette émission sont décrits par deux termes exponentiels, l'un rapide et de constante de temps $\tau_{1}$. L'autre est lent et de faible amplitude. La détermination de ce terme est peu précise mais n'affecte pas le terme le plus rapide. On trouve que $1 / \tau_{1}$ varie en fonction de la pression $\mathrm{p}_{\mathrm{Kr}}$ selon un loi parabolique dont l'expression est: $\frac{1}{\tau_{1}}=\frac{1}{\tau_{\mathrm{a} 1}}+\mathrm{k}_{23} \mathrm{P}_{\mathrm{kr}}^{2}$ 
La constante $\tau_{\mathrm{al}}$ vaut $3,85 \mu$ s et représente la durée de vie apparente de l'état $\mathrm{Kr}\left({ }^{3} \mathrm{P}_{1}\right) ; \mathbf{k}_{23}$ traduit la constante de formation de l'état moléculaire $\mathrm{Kr}_{2}\left[\mathrm{Ou}^{+}\left({ }^{3} \mathrm{P}_{1}\right)\right]$ par collisions triples.

Dans le mélange $\mathrm{Kr}$-Xe la quantité de xénon rajoutée est faible. La pression partielle de xénon est comprise entre 0,01 et 0,4 Torr. Les histogrammes expérimentaux, obtenus après des temps de stockage importants, sont également décrits par deux termes exponentiels dont le terme le plus lent est de faible amplitude. La loi d'évolution de l'inverse $1 / \tau$ du terme le plus rapide en fonction des pressions partielles des deux gaz est:

$$
\frac{1}{\tau}=\frac{1}{\tau_{\mathrm{a} 1}}+\mathrm{k}_{22}^{\prime} \mathrm{P}_{\mathrm{Xc}}+\mathrm{k}_{\mathrm{T} 2} \mathrm{P}_{\mathrm{Kr}} \mathrm{P}_{\mathrm{Xc}}+\mathrm{k}_{32} \mathrm{P}_{\mathrm{Kr}}^{2}
$$

L'émission observée derrière ce filtre traduit la disparition de l'état atomique $\mathrm{Kr}\left({ }^{3} \mathbf{P}_{1}\right)$ radiativement et collisionnellement. On met en évidence le phénomène d'emprisonnement de la transition ${ }^{3} \mathrm{P}_{1}-{ }^{1} \mathrm{~S}_{0}$, comme dans le gaz pur[11]. La constante de formation de l'état $\mathrm{Kr}_{2}\left[0 \mathrm{u}^{+}\left({ }^{3} \mathbf{P}_{1}\right)\right]$ par collisions triples homonucléaires est de $108 \mathrm{Torr}^{-2} \mathrm{~s}^{-1}$. Une réaction hétéronucléaire de formation à trois corps conduisant aux états $\mathrm{K}_{2}\left[0 \mathrm{u}^{+}\left({ }^{3} \mathrm{P}_{1}\right)\right]$ et / ou $\mathrm{Kr}^{*}-\mathrm{Xe}$ peut également être envisagée. Une réaction de transfert d'énergie à deux corps depuis l'état $\mathrm{Kr}\left({ }^{3} \mathrm{P}_{1}\right)$ sur le xénon à l'état fondamental est mise en évidence: la constante de transfert est voisine de $6,8 \cdot 10^{+4}$ Torr $^{-1} \mathrm{~s}^{-1}$.

Ces résultats montrent que, d'ores et déjà, au vu des fréquences de disparition de l'état $\operatorname{Kr}\left({ }^{3} \mathrm{P}_{1}\right)$, une deuxième voie de transfert doit être envisagée pour expliquer les résultats spectroscopiques.

\section{Références}

[1] Tanaka Y., Yoshino K.and Freeman D.E. :J.Chem. Phys., 59 (1973) 5160-5183

[2] Leichner P. K., Palmer K. F., Cook J. D. and Thieneman M.:Phys. Rev. A, 13 (1976) 1787.

[3] Wieme W. and Lenaerts J.:J.Chem. Phys., 72 (1980) 2708-2712.

[4] Th-1 Thornton G., Poliakoff E. D., Mathias E., Souyhworth S. H.,Rosenberg R. A., White M. G. and Shirley D. A.:J.Chem. Phys., 71 (1979) 133-139

[5] Millet P., Birot A.,Brunet H., Galy J., Pons-Germain B. andTeyssier J. L. -.J.Chem. Phys., 69 (1978) 92-97

[6] Salamero Y., Birot A., Brunet H., Galy J. and Millet P.:J.Chem. Phys., 80 (1984) 47744780.

[7] Berejny P., Millet P., Saissac M., and Salamero Y.J. Phys. B:At. Mol. Phys. 26 (1993) 3339-3353

[8] Freeman D. E., Yoshino K. and Tanaka Y.:J.Chem. Phys., 67 (1977) 3462-3479

[9] Castex M.C.:Thèse de Doctorat ès-Sciences, C.N.R. S., ñ AC 11038 (1973) Paris .

[10] Salamero Y.:Thèse de Doctorat ès-Sciences, $n^{\circ} 1149$ (1984) Toulouse III .

[11] Salamero Y., Kerdoussi A., Birot A., Galy J. and Millet P.:J.Phys. B, 23 (1990).35693583

[12] Mahon R., Thomas J. Mellrath, Myerscough V.P.and Koopman D.IEEE J. Quant.Elec. QE-15,n6 (1979) 444-451

[13] Bjorklund G. C.: I.E. E. E. J. Quant. Elect., 11,(1975) 287.-296 